\title{
Estudo farmacobotânico das folhas de amoreira-preta, Morus nigra L., Moraceae
}

\author{
Marina M. Padilha, Lucimara Q. Moreira, Fernanda F. Morais, Tomáz. H. Araújo, \\ Geraldo Alves-da-Silva*
}

\begin{abstract}
${ }^{1}$ Laboratório de Plantas Medicinais e Fitoterápicos, Departamento de Farmácia, Universidade Federal de Alfenas, Rua Gabriel Monteiro da Silva, 700, 37130-000 Alfenas-MG, Brasil.
\end{abstract}

\begin{abstract}
RESUMO: Morus nigra L., Moraceae, é uma espécie pertencente à família Moraceae, conhecida comumente como amoreira-preta. Na medicina popular é empregada nos casos de ondas de calor e até mesmo indicada como terapia de reposição hormonal. Possui atividade antioxidante, hipoglicemiante, antiinflamatória e antimicrobiana. O presente trabalho trata da caracterização estrutural da folha, de modo a contribuir na morfodiagnose para o controle de qualidade. Amostras do material vegetal foram seccionadas, fixadas e coradas com fucsina e toluidina. A folha apresenta estômatos anomocíticos na face abaxial, tricomas tectores, mesofilo bifacial, drusas, nervura central semi-convexa e o pecíolo exibe feixes vasculares em arco fechado.
\end{abstract}

Unitermos: Amoreira-preta, Morus nigra, Moraceae, morfodiagnose.

\begin{abstract}
Morus nigra L., Moraceae, is a species usually known as a black mulberry tree. In traditional medicine, M. nigra is used in cases of heat waves and even suitable as a therapy of hormonal replacement. This specie has antioxidant, hypoglycemic, anti-inflammatory and antimicrobial activities. This work has dealt with the structural diagnosis of the leaf, in order to contribute to its quality control. Samples of the botanical material were sectioned, fixed and stained with fuchsine and toluidine. The leaf has anomocytic stomata on the abaxial side, non-glandular trichomes, biface mesophyll, druses, semiconvex midrib and the petiole exhibits a vascular bundle in a closed arch shape.
\end{abstract}

Keywords: Black-mulberry, Morus nigra, Moraceae, structural diagnosis.

\section{INTRODUÇÃO}

Os parâmetros morfo-anatômicos possibilitam o controle botânico de qualidade de insumos farmacêuticos auxiliando a autenticidade de drogas e seus adulterantes, identificando e separando uma determinada espécie vegetal de outras. Di Stasi (1996) assinala a importância da análise morfo-anâtomica para o controle de qualidade da matériaprima vegetal na indústria farmacêutica, justificando que esta análise fornece subsídios que contribuem na padronização dos insumos, permitindo a diferenciação inclusive entre espécies botanicamente próximas. Dentre os ensaios anatômicos destacam-se os referentes a histoquímica, pois auxiliam na caracterização de drogas e no monitoramento de grupos de substâncias presentes.

A Morus nigra L., Moraceae, é uma espécie vegetal que tem sua origem na Ásia, frutificando com maior intensidade e abundância sobretudo na Ásia Menor e estando plenamente aclimatizada no Brasil (Cruz, 1979). O gênero Morus é constituído de aproximadamente 24 espécies, uma subespécie, sendo descrito pelos menos
100 variedades (Machii et al., 2000; Tutin et al., 1996). Árvore de $5 \mathrm{a} 20 \mathrm{~m}$ de altura com folhas bastante grossas, simples e alternas, cordiformes, simétricas na base, de cor verde escura, pecíolos curtos, ásperas, com dentes largos e regulares, estípulas longas, membranosas e felpudas (Morgan, 1982). As gemas são protegidas por estípulas caducas, inflorescência na forma de espiga ou sicônio. As flores são unissexuais; as masculinas monoclamídeas, isostêmones e as femininas aclamídeas, ovário súpero, bi ou unicarpelar, unilocular, uniovulado, com estigmas bífidos (Agarez et al., 1994). As infrutescências são grandes, ovaladas, negras ou vermelhas brilhantes, quase sésseis. Seus frutos são comestíveis de sabor agridoce, muito sumosas e refrescantes (Morgan, 1982). Na medicina popular as folhas da amoreira-preta têm sido indicadas para mulheres durante a menopausa. Estudos estão sendo realizados para a comprovação da ação em receptores estrogênicos (Franzotti et al., 2004). Os frutos de Morus contém compostos fenólicos que apresentaram amplo espectro de atividade bioquímica, tais como propriedades antioxidantes, antimutagênicas e anticarcinogênicas 
bem como a capacidade de modificar a expressão gênica (Nakamura et al., 2003).

Diferentes grupos de compostos químicos têm sido investigados no gênero Morus, tais como alcaloides, cumarinas, flavonoides, triterpenos e esteroides (Toshio et al., 2005; Camélia et al., 1997). Na medicina chinesa, as plantas do gênero Morus são usadas como antiinflamatório, diurético, antitussígeno, analgésico e antipirético (Nomura, 1988; Jiang, 1977). As raízes são utilizadas no tratamento de hipertensão arterial, reumatismo, problemas oculares e espasmos infantis. O fruto da amora é usado para doenças hepáticas e renais e suas folhas utilizadas para o tratamento de febre, dor de cabeça, beribéri, vômitos e dor estomacal causada pelo agente da cólera Os ramos jovens da árvore são usados para o tratamento de hipertensão e paralisia de braços e pernas (Jiang, 1977). Segundo Toshio et al. (2005), foi isolada uma substância denominada chalcomoracina de espécies de Morus, a qual apresentou considerável atividade antimicrobiana contra Staphylococcus aureus resistentes á meticilina. A potência da atividade inibitória contra o crescimento microbiano foi comparada à vancomicina.

\section{MATERIAL E MÉTODOS}

As amostras do material vegetal, Morus nigra L., Moraceae, foram coletadas de cinco plantas cultivadas no município de Alfenas-MG, Brasil, em agosto de 2007, cujas coordenadas aproximadas são $21^{\circ} 26^{\prime}$ S e $45^{\circ} 56^{\prime}$ W. A identificação botânica foi realizada pelo Prof. Dr. Geraldo Alves da Silva, responsável pelo Laboratório de Plantas Medicinais e Fitoterápicos e a exsicata foi depositada no herbário sob o nº HUNIFAL 00193.

\section{Caracterização macroscópica}

A droga, constituída de folha, foi analisada com auxílio de lupa e à vista desarmada, utilizando como instrumento de medida uma régua. Foram realizadas fotografias para documentar a caracterização (Silva \& Akisue, 2000).

\section{Caracterização microscópica}

O estudo anatômico, visando à caracterização microscópica da droga vegetal, foi realizado de acordo com as técnicas de Kraus \& Ardruin (1997) e Oliveira et al. (1991). A folha adulta foi seccionada transversalmente em micrótomo, na espessura de 10 a $20 \mu \mathrm{m}$. As secções paradérmicas foram realizadas em lâminas temporárias, com material fresco, seccionado a mão livre, e coradas com fucsina e toluidina. Todas as secções foram analisadas e fotografadas em microscópio com câmara digital.

\section{RESULTADOS}

\section{Caracterização macroscópica}

As folhas apresentaram o limbo simples, com coloração verde escuro na face ventral e coloração verde claro na face dorsal, flexível e membranácea. O limbo apresenta-se íntegro, superfície rugosa e forma oval. Em média, as folhas atingem 16,0 x 8,0 cm. A margem é serrilhada, apresentando base arredondada e ápice acuminado. A nervação é peninérvia, apresentando cor mais clara que o limbo (Figura 1).

O pecíolo apresenta coloração verde claro, torcido e curto, podendo aparecer exemplares com pecíolos em tamanho mediano. Atingem em média $9 \mathrm{~cm}$, possue superfície pilosa, inserção lateral e é biconvexo em secção transversal (Figura 1).

\section{Caracterização microscópica}

Em secção paradérmica as células epidérmicas apresentam contorno levemente ondulado e paredes finas (Figura 2). Os estômatos anomocíticos localizam-se exclusivamente na face abaxial (Figura 3) e inserem-se no mesmo nível das células circunvizinhas, podendo estar rodeados de 3, 4, 5 até 6 células. A análise possibilitou a observação de cutícula estriada na epiderme inferior e cutícula lisa na epiderme superior.

A folha em seção transversal apresenta mesofilo heterogêneo bifacial, com aproximadamente 50\% de parênquima paliçádico. As células da epiderme inferior se apresentam menores que as células da epiderme superior. No mesofilo observou-se presença de litocistos com cistólitos em forma de pêndulo ou badalo de sino (Figuras 5 e 6$)$.

Em cortes tranversais da nervura central observouse a presença de tricomas tectores simples (Figura 4). A nervura central tem secção semi-convexa (Figura 7) e a epiderme possui as mesmas características previamente descritas. Subjacente ao sistema de revestimento, notase o colênquima angular, formado por cerca de 5 a 6 camadas junto a face adaxial e 4 a 5 no lado oposto. No parênquima fundamental encontra-se um feixe vascular colateral em arco aberto. Interno ao arco formado por esse sistema vascular principal, voltado para a face adaxial, observam-se pequenos cordões de xilema e floema (Figura 8). Observou-se a presença de drusas no parênquima fundamental (Figuras 9, 10 e 11)

Observou-se também a presença de tricomas tectores simples em secções transversais do pecíolo. Este revela organização interna mostrando feixes vasculares do tipo colateral, separados por raios de parênquima fundamental (Figura 12). Apresenta epiderme uniestratificada com cutícula espessada. O colênquima é do tipo angular, e formado por aproximadamente 12 camadas, seguido de células parenquimáticas (Figura 12). 

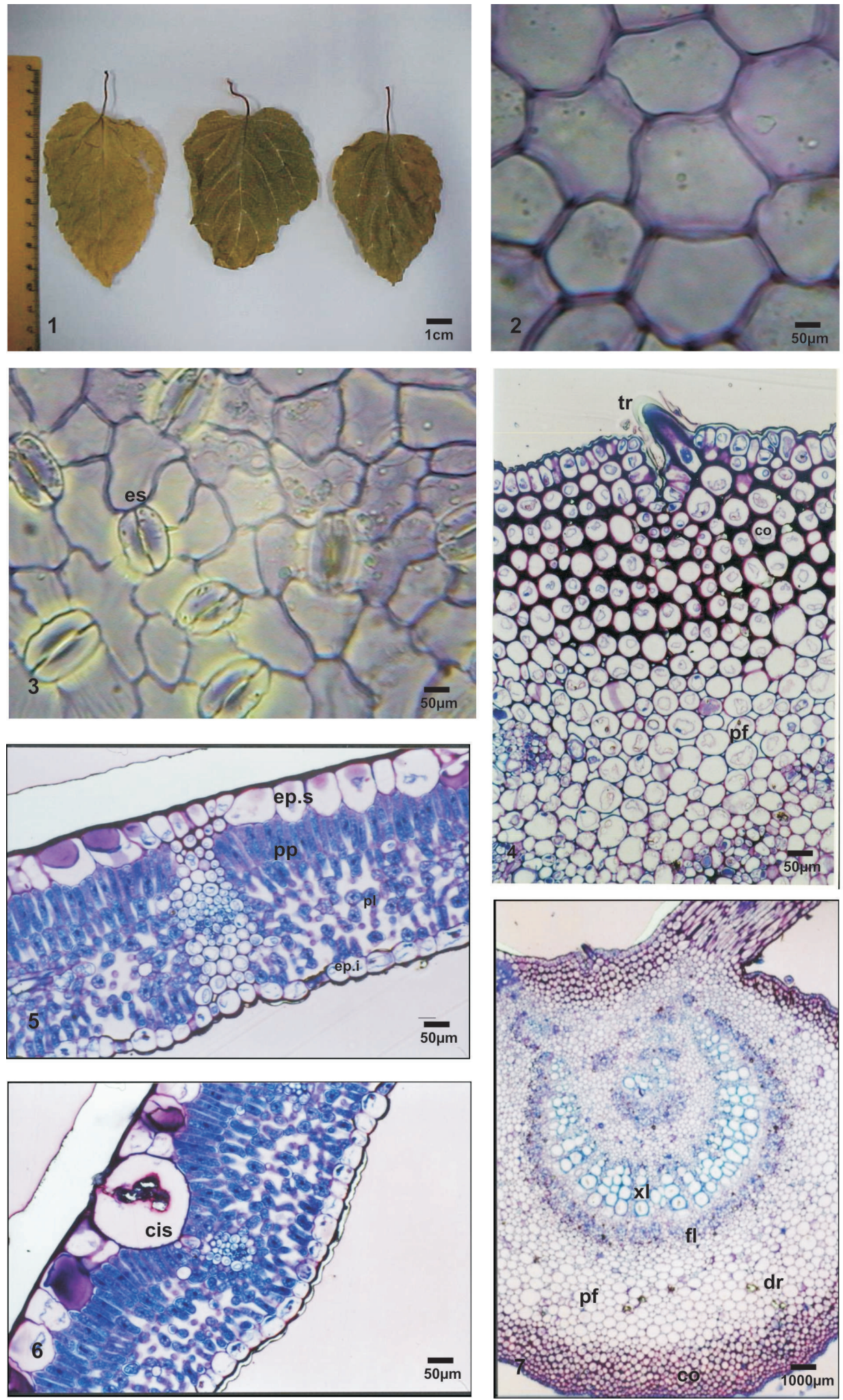

Figuras 1-7. Morus nigra L., Moraceae: 1. droga vegetal; 2. face epidérmica adaxial; 3. face epidérmica abaxial; 4. nervura central, em secção transversal, indicando a epiderme com tricoma, colênquima e parênquima fundamental; 5,6 . secção transversal do mesofilo dorsiventral; mostrando epiderme, parênquimas paliçádico e lacunoso, feixe vascular marginal e detalhe do cistólito em forma de pêndulo; 7. secção transversal da nervura central semi-convexa. cis: cistólito; co: colênquima; dr: drusas; ep.i: epiderme inferior; ep.s: epiderme superior; es: estômato; fl: floema; pf: parênquima fundamental; pl: parênquima lacunoso; pp: parênquima paliçádico; tr: tricomas; xi: xilema. 

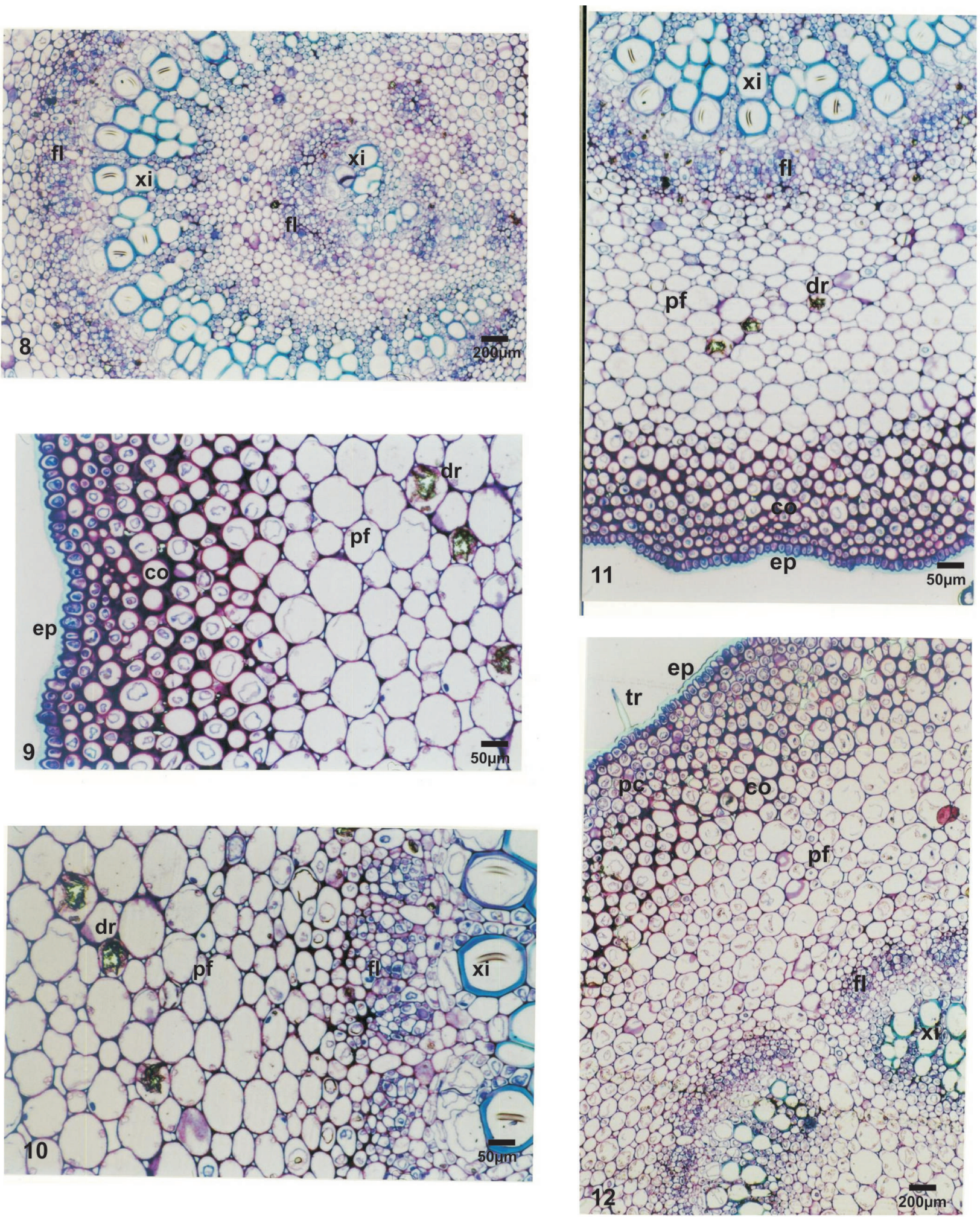

Figuras 8-12. Morus nigra L., Moraceae: 8, 9, 10 e 11. nervura central, em secção transversal, onde se notam detalhes dos feixes vasculares e drusas; 12 . pecíolo, em secção transversal, onde se evidencia feixes vasculares do tipo colateral e presença de tricomas. co: colênquima; dr: drusas; ep: epiderme; fl: floema; pc: parênquima clorofiliano; pf: parênquima fundamental; tr: tricoma; xi: xilema. 


\section{DISCUSSÃO}

Em relação à análise macroscópica da Morus nigra L., Moraceae, foi verificado que as características morfológicas da folha não destoam do gênero como um todo. Mas segundo Morgan (1982), as folhas são cordiformes e apresentam pecíolos curtos. No entanto, na espécie estudada as folhas apresentaram forma oval e pecíolo curto, podendo aparecer exemplares com pecíolo em tamanho mediano.

Os caracteres da epiderme foliar são discordantes com a descrição de Sonibare et al. (2006), quanto ao formato das células epidérmicas, que segundo os autores são retangulares, e no presente estudo observou-se que estas células apresentam contorno levemente ondulado.

Diferentemente ainda foi observado por Sonibare et al. (2006), que algumas espécies da família Moraceae apresentam 2 a 3 camadas de células no parênquima paliçádico. Embora tenha observado a presença do parênquima paliçádico no mesofilo da espécie estudada, este se encontra com o mesmo número de camadas do parênquima lacunoso (torno de $50 \%$ em cada).

$\mathrm{Na}$ epiderme deve ser destacada a presença de tricomas tectores que concordam com a descrição de Sonibare et al. (2006) e Biasiolo et al. (2004). Outra característica relevante na anatomia foliar desta espécie, que pode ser útil em sua diagnose como insumo farmacêutico, é que as células da epiderme inferior apresentam-se menores que na epiderme superior.

A análise microscópica revelou a ocorrência de estômatos do tipo anomocítico, localizando-se exclusivamente na face abaxial.

Metcalfe \& Chalk (1957) caracterizaram a anatomia foliar de plantas da família Moraceae, tendo constatado na epiderme de Morus sp., a presença de cistólitos. Katsumata (1971) e Fujita \& Uchikawa (1986) também observaram idioblastos de cistólitos na epiderme foliar de amoreira. Segundo Mauseth (1988), os cristais podem ocorrer em diferentes órgãos das plantas e em vários formatos, tais como drusas, cristais prismáticos $\mathrm{e}$ estilóides. Entretanto cristais de carbonato de cálcio são encontrados apenas em poucas famílias como Moraceae, Urticaceae e Acanthaceae. O cistólito de carbonato de cálcio é localizado no litocisto e ocorrem principalmente na epiderme das folhas. Existe um número crescente de registros revelando a formação de cristais de cálcio que tanto podem ser de oxalato (Franceschi \& Horner, 1980; Borchert, 1986; Doaigey, 1991; Kuo-Huang et al., 1994) como cristais de carbonato de cálcio (Watt et al., 1987; Yu \& Li, 1990; Kuo-Huang \& Yen, 1996). Apenas poucos relatos descrevem a presença de ambos cristais em uma mesma espécie (Fahn, 1990) e tem correspondência com o verificado neste estudo,onde foi observado apenas cistólitos com inclusões de carbonato de cálcio.

\section{CONCLUSÕES}

Vários parâmetros morfo-anatômicos listados neste trabalho são significativos na diagnose da $M$. nigra como insumo farmacêutico. Como características relevantes no controle botânico de qualidade desta planta destaca-se a presença de estômatos anomocíticos exclusivamente na face abaxial, feixe vascular colateral em arco aberto, mesofilo heterogêneo, drusas no parênquima fundamental, tricomas tectores e cistólitos. Todavia, devem ser considerados em conjunto levando-se em conta que alguns caracteres são comuns a outros representantes do gênero e isoladamente não apresentam valor taxonômico para sua diferenciação, uma vez que espécies em grupos muito próximos apresentam caracteres estruturais e compostos químicos em comum.

\section{AGRADECIMENTOS}

Os autores agradecem a CAPES e PIBIC/CNPq pelas bolsas concedidas.

\section{REFERÊNCIAS}

Agarez FV, Cézio P, Cecília MR 1994. Botânica: Taxonomia, morfologia e reprodução das angiospermas. 2 ed. Rio de Janeiro: Âmbito Cultural, $256 \mathrm{p}$.

Biasiolo M, Canal MTD, Tornadore N 2004. Micromorphological characterization of tem mulberry cultivars (Morus spp). Econ Bot 58: 639- 646.

Borchert R 1986. Calcium acetate induces calcium uptake and formation of calcium oxalate crystals in isolated leaflets of Gleditsia triacanthos L. Planta 168: 571-578.

Cruz GL 1979. Dicionário de plantas úteis no Brasil. Rio de Janeiro: Civilização Brasileira. 599 p.

Di Stasi LC 1996. Plantas Medicinais: arte e ciência. São Paulo:Unesp. 230 p.

Doaigey AR 1991. Occurrence, type, and location of calcium oxalate crystals in leaves and stem of 16 species of poisonous plants. Am J Bot 78: 1608-1616.

Fahn A 1990. Plant Anatomy. 4th ed. Pergamon Press. Oxford.

Franceschi VR,. Horner HT Jr 1980. Calcium oxalate crystals in plants. Bot Rev 46: 361.427.

Franzotti EM, Melo SRO, Neves FAR, Silveira D, Simeoni LA 2004. Ação de extratos de Morus nigra em receptores de estrogênio. XVIII Simpósio de Plantas Medicinais do Brasil. Manaus, Brasil.

Fujita H, Uchikawa C 1986. Electron microscopical study of mulberry with special reference to the identification of cultivars. In: Kitaura K (Ed.). Development of new technology for identification and classification of tree crops and ornamentals. Yatabe-cho, Tsukuba-gun, Ibaraki-ken, Japan: Fruit Tree Research Station, Ministry of Agriculture Forestry and Fisheries. p. 25-29.

Jiang Su Xin Yi Xue Yuan 1977. (New Jiang-Su Medical School working party), Zhong Yao Da Ci Dian (Encyclopaedia 
of Chinese crude drugs), Shangai: Shanghai Ke Xue Ji Shu Chu Ban She (Shanghai Science and techonology Publisher).

Katsumata F 1971. Shape of idioblasts in mulberry leaves with special reference to the classification of mulberry trees. $J$ Sericultural Sci Japan 40: 313-322.

Kraus JE, Arduin M 1997. Manual Básico de Métodos em Morfologia Vegetal. In: Seropédica. Rio de Janeiro: EDUR, p. 198.

Kuo-Huang LL, Sheue CR, Yang YP, Chiang SHT 1994. Calcium oxalate crystals in some aquatic angiosperms of Taiwan. Bot Bull Acad Sinica 34: 179-188.

Kuo-Huang LL, Yen TB 1996. The development of lithocysts in the leaves and sepals of Justicia procumbens L. Taiwania 41: 17- 26.

Machii H, Koyama A, Yamanouchi H 2000. FAO Eletronic Conference: Mulberry for animal production. Disponível em http://www.fao.org/livestock/agap/frg/mulberry.

Mauseth JD 1988. Plant anatomy. Menlo Park: Benjamim Cummings.

Metcalfe CR, Chalk L 1957. Anatomy of the dicotyledons. Oxford: Clarendon Press 1: 1259-1271.

Morgan R 1982. Enciclopédia das ervas e Plantas Medicinais. São Paulo: Hemus editora. 555 p.

Nakamura Y, Watanabe S, Miyake N, Kohno H, Osawa T 2003. Dihydrochalcones: evaluation as novel radical scavenging antioxidants. J Agr Food Chem 51: 3309-3312.

Nomura T 1988. Phenolic compounds of the mulberry tree and related plants. In: Herz W, Grisebach H, Kiurby GW, Tamm Ch, eds. Progress in the chemistry of organic natural products 53. Vienna: Springer Publishing. p. 87.

Oliveira F, Akissue G, Akissue MK 1991. Farmacognosia. São Paulo: Atheneu, p. 412.

Silva GA, Akisue G 2000. Morfodiagnose de Cissus sicyoides L. (insulina vegetal). Lecta 15: 63-97.

Sonibare MA, Jaycola, Egunyomi A 2006. Comparative leaf anatomy of Ficus Linn. Species (Moraceae) from Nigeria. J Appl Sci 6: 3016- 3025.

Toshio F, Kiyoshi K, Sumio T 2005. Antimicrobial activity of 2-arylbenzofurans from Morus species against methicillin-resistant Staphylococcus aureus. Fitoterapia 76: 708-711.

Tutin GT, Burges NA, Chater AO, Edmondson JR, Heywood VH, Moore DM, Valentine DH, Walters SM, Webb DA. 1996. Morus L. In Flora Europa. Psilotaceae to Platanaceae. vol 1. Australia: Cambrigde University Press.

Watt WM, Morrell CK, Smith DL, Steer MW 1987. Cystolith development and structure in Pilea cadierei (Urticaceae). Ann Bot-London 60: 71-84.

Yu FG, Li ZI 1990. Anatomy of the lithocyst in the epidermis of leaf in Broussonetia papyrifera. Acta Bot Sin 33: 249255 